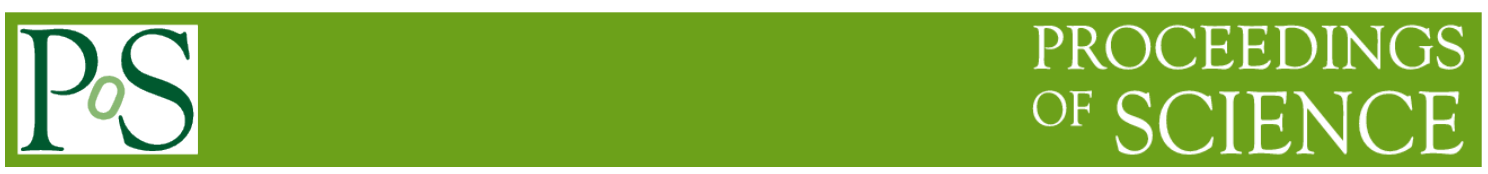

\title{
Towards Nuclear Spin Polarization of DT
}

\author{
J.-P. Didelez ${ }^{1}$ \\ IPN, CNRS/IN2P3 \& Université Paris-Sud (UMR-CNRS 8608) \\ Bât. 100, F-91406 ORSAY, France \\ E-mail: didelezlipno.in2p3.fr \\ C. Deutsch \\ LPGP, Université Paris-Sud (UMR-CNRS 8578) \\ Bât. 210, F-91405 ORSAY, France \\ E-mail: claude.deutscheu-psud.fr
}

\begin{abstract}
Recently, we have proposed an experiment to test the persistence of the polarization in a fusion process, using a powerful laser hitting a polarized $H D$ target. In the present contribution, we first examine new possibilities towards this goal, in view of recent developments of new polarized $D$ samples presented in this conference. We then advocate that sizeable amounts of polarized $D T$ fuel could be obtained by the Nuclear Dynamic Polarization (DNP) of DT molecules, if a successful protocol for the DNP of the $H D$ analog isotopic molecule could be established. We suggest that a revival of an early $H D$ DNP experiment performed more than forty years ago, should be undertaken, taking advantage of detailed polarized $H D$ properties revealed by thoroughly repeated static polarization of $H D$ targets during the last ten years.
\end{abstract}

${ }^{1}$ Speaker 
23rd International Spin Physics Symposium - SPIN2018 -

10-14 September, 2018

Ferrara, Italy 


\section{Introduction}

It is accepted that the parallel polarization of $D$ and $T$ nuclei should increase their reactivity when used as fuel material in fusion processes induced either by magnetic or by inertial confinement, because the fusion reaction goes mainly through the excitation of a resonant ${ }^{5} \mathrm{He} 3 / 2^{+}$intermediate state. In addition, the reaction products would be emitted with a $\sin ^{2} \theta$ angular distribution, by reference to the polarization axis. This can be very useful to reduce damage or activation of costly equipments. The question is to know if the polarization will persist in dense and hot plasmas as anticipated from theoretical considerations, both for Magnetic Confinement Fusion (MCF) [1] and Inertial Confinement Fusion (ICF) [2]. The persistence of the polarization in a fusion process could be tested using a powerful laser hitting a polarized $H D$ target. The polarized deuterons accelerated in the plasma generated by the laser can fuse, according to the reaction: $\left(D+D \rightarrow{ }^{3} \mathrm{He}+n\right)$. The angular distribution of the emitted neutrons and the change in the corresponding total cross section are signatures to estimate the polarization persistence [3].

In a more recent paper [4], we have discussed the experimental difficulties in using such a polarized $H D$ target produced by the static polarization method "Brute Force" (BF) which is costly and takes long time, in addition to the sophisticated cryogenic holding equipment necessary to maintain the target on a laser beam line, as well as the lack of a clean flat surface of the solid $H D$ on which to adjust the laser focusing. The main difficulty comes from the fact that the polarized material must be contained in a thin Mylar bag, offering to the laser only a small window-less surface. But so far, the BF method was almost the only one to get rather pure polarized $D$ samples. Recent developments reported in this symposium can produce much better polarized targets either by using hyper-polarized molecules from Atomic Beam Sources (ABS) [5] or highly nuclear-spin-polarized deuterium atoms from UV photodissociation [6,7]. We shall briefely discuss below, how the corresponding experiments could be done.

\section{New polarized $D 2$ samples}

Polarized atoms from an ABS can recombine to molecules on a cold surface inside a dedicated apparatus [5]. During this process, it is expected that a large fraction of the initial polarization (close to $100 \%$ ) could be preserved. This would allow to produce a thin coating of solid $D_{2}$, which could be used as windowless target instead of the bulky $H D$ target in our initial proposal [3]. Depending on the ABS intensity and the efficiency of the whole solidification process, a $1 \mathrm{~cm}^{2}$ target of a few $\mu \mathrm{m}$ thickness (enough for a laser-D2 interaction) could be produced within hours, to be compared with months of production for a polarized $H D$ target by BF with final lower $D$ polarization rates [8].

Such a target would allow to repeat the Pretzler experiment [9] in quasi identical conditions except for the polarization of $D$ nuclei. It should be noted that in Ref. [9], a prepulse of the laser is used to to generate a preplasma with a scale length of $30 \mu \mathrm{m}$ when the main pulse arrives after $300 \mathrm{ps}$. The authors note that without such a prepulse, no $D D$ fusion where observed, so that the fusion reactions take place inside the preplasma.

This suggests that an other kind of polarized target could also be used. It has been shown in this symposium [7] how laser can be used to create polarized deuterium samples at nearly 
atmospheric pressure $\left(10^{19} \mathrm{~cm}^{-3}\right)$. The exact experimental conditions of [9] $\left(\mathrm{n}_{\mathrm{e}} \sim 10^{21} \mathrm{~cm}^{-3}\right)$ are not readily realized, but intensive numerical simulations might tailor the proper choice of the various experimental parameters to get the best fusion rate. It is likely that with modern ultrashort lasers, even with moderate pulse energies, some fusion will occur allowing to perform a significant exepriment within a reasonable time.

It is worth noting that the initial idea was that in the plasma created by the laser, polarized deuterons would be heated enough to undergo mutual thermal fusion. However, it is unlikely that thermal fusion alone could account for the observed rate of neutron production as seen in previous exepriments. More likely, some deuterons are accelerated in the plasma by the laser plasma interaction and those accelerated ions act as a beam to generate nuclear reactions with colder deuterons in the plasma. Thermal fusion dominance would probably require much more energetic laser pulses!

\section{Nuclear spin polarization of solid Hydrogen}

The Hydrogen family comprises 6 members: 3 homonuclear ones $\left(H_{2}, D_{2}\right.$ and $\left.T_{2}\right), 3$ heteronuclear ones ( $H D, H T$ and $D T$ ); among them 3 are radioactive ( $H T, D T$ and $T_{2}$ ). Due to symmetry restrictions of the wave function, the polarization of homonuclear molecules is complicated by metastables states producing at low temperature 2 coexisting configurations with different polarizabilities. As an example, the polarization of $\mathrm{H}_{2}$ at low temperature in its ortho metastable configuration ( 2 nuclear spins aligned) could be attempted, however the equilibrium configuration at low temperature is the para one ( 2 nuclear spins antiparallel), so that in a few hours, by decay of the ortho to the para form, the $H_{2}$ molecules lose their polarization. Therefore, in the last 10 years, the BF static polarization of $H D$ has been pursued, essentially because the hetero $H D$, being formed of a fermion and a boson, has no symmetry restriction of its total wave function [8]. However, to reach sizable polarization rates by BF, it is necessary to go down to very low temperatures and high magnetic fields, namely dilution refrigerators and supraconducting coils which require heavy equipments. For example, at $10 \mathrm{mK}$ and 17 Tesla, the proton polarization can reach $\sim 90 \%$, while the deuteron vector polarization is only $\sim 33 \%$ due to the lower deuteron magnetic moment. Reaching low temperatures requires some time and allowing the contaminant homonuclear species decay to their magnetically inactive forms takes more than a month. Accordingly, even at moderate low temperatures $(4 \mathrm{~K})$ and weak magnetic fields (1 T), relaxation times can become very long [8].

The $D T$ has the most interesting Hydrogen configuration, because it contains both the $D$ and $T$ nuclei necessary for nuclear fusion. Unfortunately, the BF method cannot be applied to $D T$, because the heat generated by the Tritium radioactivity does not allow to reach very low temperatures. The power of dilution refrigerators at $10 \mathrm{mK}$, is in the range of $\mu \mathrm{W}$, while the Tritium radioactivity produces a heat in the range of $\mathrm{W} / \mathrm{mol}$. For the DNP, temperatures around $1-4 \mathrm{~K}$ are sufficient and refrigerators in the range of a few $\mathrm{W}$ powers can be built. Since $H D$ and $D T$ have very similar magnetic properties, it is convenient to optimize the DNP protocol on $H D$. So far, the results onbtained by the DNP of $H D$ were not encouraging. The low polarization rates (less than 5\%) observed, could have been limited by significant amounts of $\mathrm{H}_{2}$ and $\mathrm{D}_{2}$ impurities [10]. Fig. 1 shows a schematic picture of the DNP process. The high polarization of electrons is transferred to the nuclear spins by RF transitions. The key points to get high polarization rates are: i) well resolved states so that RF transitions are connected to well defined 
hyperfine states [11], ii) electron spin-lattice relaxation times $T_{1 \mathrm{e}}$ much shorter than the nuclear relaxation ones $\mathrm{T}_{1 \mathrm{~N}}$. It turns out that for Hydrogen, $\mathrm{T}_{1 \mathrm{e}}$ is unusually long.

\section{Dynamic Polarization of HD or DT}

Added free electrons. For $\mathrm{B}=2.5 \mathrm{~T}$ and $\mathrm{T}=1 \mathrm{~K}$, $\mathrm{e}^{-}$polarization $=92 \%$

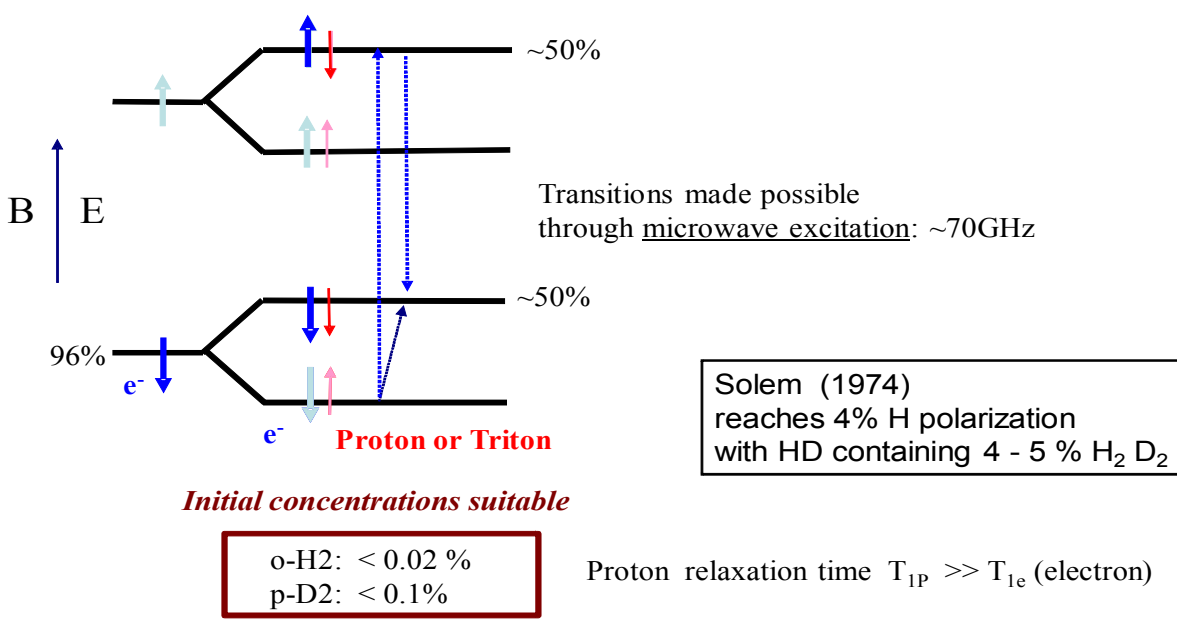

Fig. 1: Sketch of the DNP process, according to the solid state effect. The polarization of unpaired electrons, is transferred to the nuclei by RF transition between hyperfine states. If the $\mathrm{T}_{1 \mathrm{e}}$ is much shorter than the $\mathrm{T}_{1 \mathrm{~N}}$, electrons undergo spin flip rapidly, while nuclear spins keep their orientation. By repeating this process, nearly all the electron polarization can be tranferred to the nuclei.

To shorten $\mathrm{T}_{1 \mathrm{e}}$ Solem [10] added to the $H D$ a small fraction $\left(10^{-3}\right)$ of paramagnetic $O_{2}$, which shortened drastically $\mathrm{T}_{1 \mathrm{e}}$ but at the same time broadened the hyperfine states, as shown by the resulting Electron Paramagnetic Resonance (EPR) spectra measured by Solem himself. For pure $H D$, the EPR sectra show well resolved hyperfine states, both for proton and deuteron after addind $\mathrm{O}_{2}$, the states linked to the protons are significantly broadened, while those from the deuterons are severely smeared. No wonder that the deuteron polarization could hardly be build up! Pure $H D$ samples are now available with extra long $\mathrm{T}_{1 \mathrm{~N}}$. As shown in Ref. [12], $\mathrm{T}_{1 \mathrm{~N}}$ can be increased almost at will by using higly distilled $H D$ gas and ageing it to let the ortho- $\mathrm{H}_{2}$ and para- $D_{2}$ impurities decay to their magnetic inactive configurations. Compared to the $\mathrm{T}_{1 \mathrm{e}}$ in the range of seconds as mentionned by Solem, $\mathrm{T}_{1 \mathrm{~N}}$ in the range of hours have been achieved [12]. A suitable density of unpaired electrons coud be generated by intensive irradiation. Recent exposition of polarized $H D$ samples to electrons have shown some polarization loss but no significant reduction of $\mathrm{T}_{1 \mathrm{~N}}$ [13]. It is obvious that the Solem investigation of the DNP of $H D$ should be repeated, using recent expertise on the $H D$ properties. An optimum DNP protocol could be established and extended to $D_{2}$ and $D T$ for polarized fusion experiments.

\section{Conclusion}

It has been shown that the experiment initially proposed to test the persistence of the polarization in a fusion process, could be done in much better experimental conditions, using presently available polarized window-less $D_{2}$ samples. Although the related polarization 
methods from ABS or UV dissociation have a great potential, it is not clear yet how they can produce enough polarized fuel for fusion.

A fusion reactor like ITER, with a power of $500 \mathrm{MW}$ would need at least $1 \mathrm{~mol} / \mathrm{hour}$ of $D T$, assuming $100 \%$ efficiency, which will not be by far the case! Therefore, it is highly desirable to find a way to polarize sizeable amounts of $D T$. It has been advocated that the DNP protocol of solid $H D$, if successful after repeating early experiments in presently much better conditions, could be extended to $D T$ and even $D_{2}$. It should be noted that sizeable quantities of polarized material are already produced by DNP for nuclear physics experiments: the COMPASS polarized target at CERN is able to polarize $50 \mathrm{~mol}$ of material within a single polarization cycle [14].

\section{References}

[1] R.M, Kulsrud et al., Fusion reactor plasmas with polarized nuclei. Phys. Rev. Lett. 49 (1982) 1248.

[2] R.M. More, Nuclear spin-polarized fuel for inertial fusion. Phys. Rev. Lett. 51 (1983) 396.

[3] J.P Didelez and C. Deutsch, Persistence of the polarization in a fusion process. Laser Part. Beams 29 (2011) 169.

[4] J.P Didelez and C. Deutsch, DD fusion from a polarized HD target. Laser Part. Beams 33 (2015) 211.

[5] R. Engels et al., Production of hyperpolarized H2 molecules from H atoms in gas-storage cells. Phys. Rev. Lett. 115 (2015) 11307. Production and storage of polarized H2,D2 and HD molecules. Contribution to this Symposium, ID : 37.

[6] D. Sofikitis et al., Highly nuclear-spin-polarized deuterium atoms from the UV photodissociation of deuterium iodide. Phys. Rev. Lett. 118 (2017) 233401. Laser preparation of intense beams of vectorially polarized protons and tensorially polarized deuterons via molecular quantum beats. Contribution to this Symposium, ID : 27.

[7] T. P. Rakitsis, Optical excitation of molecules for Spin-Polarized Nuclear Fusion. Contribution to this Symposium, ID : 119.

[8] J. P. Didelez, A polarized HD target for nuclear physics experiments with real photons. Nucl. Phys. News 4 (1994) 10.

[9] G. Pretzler et al., Neutron production by $200 \mathrm{~mJ}$ ultrashort laser pulses. Phys. Rev. E 58 (1998) 1165.

[10] J.C. Solem, Dynamic polarization of protons and deuterons in solid deuteron hydride. Nucl. Inst. and Meth. 117 (1974) 477.

[11] J.C. Solem and G. A. Rebka, EPR of atoms and radicals in radiation-damaged $\mathrm{H}_{2}$ and $\mathrm{HD}$. Phys. Rev. Lett. 21 (1968) 19.

[12] S. Bouchigny et al., Distillation of HD gas and measurement of of spin-lattice relaxation times. Nucl. Inst. and Meth. A 577 (2007) 320.

[13] A. Sandorfi, Potential use of solid frozen-spin HD targets with electron beams. Contribution to this Symposium, ID : 118

[14] J. Ball et al., First results of the large COMPASS ${ }^{6}$ LiD polarized target. Nucl. Inst. and Meth. A 498 (2003) 101. F. Kunne, private communication. 